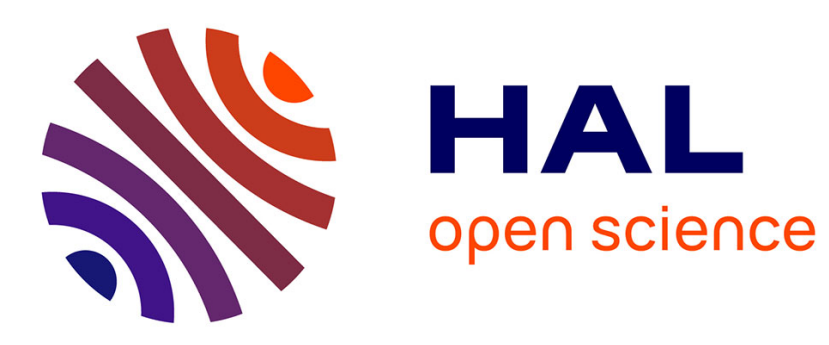

\title{
Self-employment, start-up incentives and political ideology
}

\author{
Stefano Horst Baruffaldi, Marianna Marino, Pierpaolo Parrotta
}

\section{To cite this version:}

Stefano Horst Baruffaldi, Marianna Marino, Pierpaolo Parrotta. Self-employment, start-up incentives and political ideology. Applied Economics Letters, 2016, 23 (4), pp.250-254. 10.1080/13504851.2015.1068914 . hal-01512864

\section{HAL Id: hal-01512864 \\ https://hal.science/hal-01512864}

Submitted on 14 Jun 2018

HAL is a multi-disciplinary open access archive for the deposit and dissemination of scientific research documents, whether they are published or not. The documents may come from teaching and research institutions in France or abroad, or from public or private research centers.
L'archive ouverte pluridisciplinaire HAL, est destinée au dépôt et à la diffusion de documents scientifiques de niveau recherche, publiés ou non, émanant des établissements d'enseignement et de recherche français ou étrangers, des laboratoires publics ou privés. 


\title{
Self-employment, Start-up Incentives and Political Ideology
}

\author{
Stefano Horst Baruffaldi* $\quad$ Marianna Marino ${ }^{\dagger} \quad$ Pierpaolo Parrotta $^{\ddagger}$
}

\begin{abstract}
We find evidence that public start-up incentives promote self-employment. This public policy seems to affect more women than men.
\end{abstract}

JEL Classification: L26, J21, J58.

Keywords: Self-employment, start-up incentives, political ideology.

\footnotetext{
*École Polytechnique Fédérale de Lausanne, Chair in Economics and Management of Innovation, Station 5 CH-1015 Lausanne, Switzerland. E-mail: stefano.baruffaldi@epfl.ch.

${ }^{\dagger}$ ICN Business School, Department of Strategy and Innovation, 3 Place Edouard Branly, 50070 Metz Technopole, France and Bureau d'Économie Théorique et Appliquée (BETA), UMR CNRS 7522, Université de Lorraine, France. E-mail: marianna.marino@icn-groupe.fr

${ }^{\ddagger}$ Corresponding author, Department of Economics, School of Business and Economics, Maastricht University, 6200 MD Maastricht, Netherlands. E-mail: p.parrotta@maastrichtuniversity.nl.
} 


\section{Introduction}

Economic theory suggests that entrepreneurial activity, including self-employment or business ownership, which is the most commonly used condition to identify an entrepreneur, propels economic growth by enhancing productivity and innovation (see van Praag and Versloot, 2007). Nonetheless, self employment rates have been decreasing and a significant gender gap in self-employment rates has been emerging over the last decades in several OECD countries (OECD, 2011).

Previous studies have analysed the determinants of self-employment, focusing mainly on the role of individuals' economic incentives (e.g., Lazear and Moore, 1984), parental backgrounds (e.g., Marino and Parrotta, 2015), individual living (e.g., Blanchflower, 2000) or working (e.g., Marino et al, 2012) environments, and macroeconomic conditions (e.g., Parker et al., 2012). However, to the best of our knowledge, there is no empirical evidence concerning the effects of public start-up incentives on self-employment rates or on the ratio between female and male self-employment rates.

Our aim is to fill this gap using data for a panel of 20 OECD countries over the period 1990-2007. We implement a linear model estimation approach, controlling for a set of labour market policies and characteristics as well as allowing for country and year fixed effects to control for both unobserved heterogeneity and global economic trends. However, because public support for start-ups likely responds to economic contingencies, we adopt an IV estimation strategy exploiting voter past political ideology as an instrument for the GDP share of public expenditure for start-up incentives.

We find evidence that start-up incentives favour self-employment, especially among women. Specifically, we find that a $0.1 \%$ (or approximately 4 standard deviation) increase in the share of GDP devoted to start-up incentive expenditures increases the self-employment rate and the female to male self employment rate ratio by $0.18 \%$ and $0.22 \%$, respectively. These findings suggest that public support for start-ups is an effective policy tool to promote both self-employment and women's engagement in entrepreneurial activities.

The remainder of this paper is presented in the following order: data, estimation strategy, results and conclusions.

\section{Data}

Combining information from the OECD Labour Market Statistics, World Development Indicators (WDI), Manifesto Research Group (MRG) Project, and Institutional Characteristics of Trade Unions, Wage Setting, State Intervention and Social Pacts (ICTWSS) databases, we built a nearly balanced sample of 20 countries over a period of 18 years (1990-2007). We restrict the sample to those countries for which all relevant data are 
available over the sample period: Australia, Austria, Belgium, Canada, Denmark, Finland, France, Germany, Greece, Ireland, Italy, Japan, the Netherlands, New Zealand, Norway, Portugal, Spain, Sweden, the United Kingdom, and the United States. Furthermore, to exclude the pervasive effects of the Great Recession (20082010), we do not consider the most recent years when most countries increased their shares of public spending on start-up incentives.

Table 1 reports the descriptions, sources and descriptive statistics for our variables. The average selfemployment rate is approximately $2.7 \%$, and the average proportion of females who are self-employed is two-thirds of their male counterparts. The median voter's ideology is neither left nor right wing. On average, start-up incentives represent approximately 0.02

\section{$3 \quad$ Estimation strategy}

To investigate the effect of startup incentives on self-employment rates and on the ratio between female and male self-employment, we perform the following linear regression approach:

$$
y_{i t}=\gamma \text { StartupIncentives }_{i t}+X_{i t}^{\prime} \beta+u_{i}+\eta_{t}+\varepsilon_{i t}
$$

$y_{i t}$ indicates either the self-employment rate or female vs male self-employment, where the latter is defined as ratio between female and male self-employment rates. $X_{i t}^{\prime}$ includes an extensive set of control variables on labour market policies and labour market characteristics, and $u_{i}$ and $\eta_{t}$ are country and time fixed effects, respectively. For regressions in which female vs male self-employment is the dependent variable, we include both the female and male unemployment rates, rather than only the overall unemployment rate.

As public policies might respond to idiosyncratic economic conditions and expectations, we implement an instrumental variable strategy based on the assumption that past political ideology is an exogenous determinant of our variables of interest. Specifically, we compute the median voter's ideological position as in Kim and Fording (2001). In addition, because simultaneity between present changes in political ideology and economic conditions may still bias our analyses, we use a 5-year moving average, MA(5), of the contemporaneous political ideology indicator to allow for a lagged effect of political ideology on the variable of interest.

\section{Results}

Table 2 reports the results of both OLS (with country and year fixed effects) and FE (within effects) regression models with the self-employment rate as the dependent variable. We find that the parameter of interest is 
significant and robust to the inclusion of controls for labour market policies and labour market characteristics. We observe that a $0.1 \%$ (or approximately 4 standard deviation) increase in the GDP share of startup incentive expenditure is related to a $0.08 \%$ increase in the self-employment rate. Similarly, Table 3 reports the results for the relation between start-up incentives and female to male self-employment. Start-up incentives seem to favour female over male self-employment, although this parameter is not significant across all specifications.

Table 4 reports the results of our IV estimation strategy. The first stage regression shows the strength of our instrumental variable, the median voter's ideological position, on each variable of interest (i.e., the F-test on the excluded instrument scores are largely above the reference threshold of 10). Right parties seem to devote more resources to entrepreneurship than do left parties.

The second stage estimates confirm and reinforce the FE results: The parameters on our key variable indicate a sizable and significant impact on both dependent variables. We find that a $0.1 \%$ (or approximately 4 standard deviation) increase in the GDP share of start-up incentive expenditure increases the self-employment rate and the female to male self-employment rate ratio by $0.18 \%$ and $0.22 \%$, respectively.

\section{Conclusions}

We find evidence that start-up incentives promote self-employment, especially among women. Our findings show that political ideology influences the extent to which a country resorts to start-up incentives and that start-up incentive policies can effectively stimulate entrepreneurial activity. 


\section{References}

[1] Blanchflower, D.G., 2000. Self-employment in OECD countries. Labour Economics 7, 471- 505.

[2] Kim, H., and Fording, R. C., 2001. Does tactical voting matter? The political impact of tactical voting in recent British elections. Comparative Political Studies, 34(3), 294-311.

[3] Lazear, E.P., Moore, R.L., 1984. Incentives, productivity, and labor contracts. Quarterly Journal of Economics, 99(2), 275-296.

[4] Marino, M., and Parrotta, P., 2015. Self-employment: does parental cultural diversity matter?. Applied Economics Letters, (ahead-of-print), 1-8.

[5] Marino, M., Parrotta, P., and Pozzoli, D., 2012. Does labor diversity promote entrepreneurship?. Economics Letters, 116(1), 15-19.

[6] OECD, 2007. OECD System of Unit Labour Cost Indicators, OECD, Paris.

[7] OECD, 2011. "Self-employment", in OECD Factbook 2011-2012: Economic, Environmental and Social Statistics, OECD Publishing. http://dx.doi.org/10.1787/factbook-2011-61-en

[8] Parker, S. C., Congregado, E., and Golpe, A. A., 2012. Is entrepreneurship a leading or lagging indicator of the business cycle? Evidence from UK self-employment data. International Small Business Journal, 30(7), 736-753.

[9] Van Praag, C. M., and Versloot, P. H., 2007. What is the value of entrepreneurship? A review of recent research. Small business economics, 29(4), 351-382. 
Table 1: Descriptive statistics

\begin{tabular}{|c|c|c|c|c|}
\hline & Mean & Standard deviation & Min & Max \\
\hline \multicolumn{5}{|l|}{ Dependent variable } \\
\hline Self-employment rate (\% of civilian employment, OECD source) & 2.697 & 0.439 & 1.96 & 3.865 \\
\hline Female vs male self-employment (female to male self-employment rate ratio, WDI source) & 0.668 & 0.217 & 0.289 & 1.45 \\
\hline \multicolumn{5}{|l|}{ Key variables } \\
\hline Start-up incentives (\% of GDP, OECD source) & 0.019 & 0.028 & 0 & 0.19 \\
\hline Median voter's ideology index MA(5) (á la Kim and Fording (2001), MRG Project source) & 0.003 & 0.167 & -0.427 & 0.516 \\
\hline \multicolumn{5}{|l|}{ Labour market policies } \\
\hline Employment rehabilitation (\% of GDP, OECD source) & 0.102 & 0.15 & 0 & 0.62 \\
\hline Job creation ( $\%$ of GDP, OECD source) & 0.125 & 0.155 & 0 & 0.64 \\
\hline Training ( $\%$ of GDP, OECD source) & 0.252 & 0.193 & 0.01 & 1.06 \\
\hline Employment incentives ( $\%$ of GDP, OECD source) & 0.118 & 0.148 & 0 & 0.88 \\
\hline Early retirement ( $\%$ of GDP, OECD source) & 0.16 & 0.275 & 0 & 1.19 \\
\hline Out-of-work support (\% of GDP, OECD source) & 1.276 & 0.848 & 0.16 & 4.79 \\
\hline \multicolumn{5}{|l|}{ Labour market characteristics } \\
\hline Union coverage (\% values, ICTWSS source) & 67.163 & 25.994 & 13.1 & 99 \\
\hline Union density (\% values, ICTWSS source) & 36.737 & 20.728 & 7.544 & 83.863 \\
\hline Unit Labour Cost (index OECD base year $2005=100$ ) & 2.348 & 3.154 & -5.24 & 23.407 \\
\hline Unemployment rate (\% of labour force, OECD source) & 7.632 & 3.579 & 1.806 & 24.171 \\
\hline Female unemployment rate ( $\%$ of female labour force, WDI source) & 8.602 & 4.999 & 1.825 & 31.625 \\
\hline Male unemployment rate (\% of male labour force, WDI source) & 6.995 & 3.122 & 1.81 & 19.69 \\
\hline Employment protection legislation (regular contract, OECD version 1) & 2.149 & 0.934 & 0.257 & 4.833 \\
\hline Employment protection legislation (temporary contract, OECD version 1 ) & 1.91 & 1.391 & 0.25 & 4.875 \\
\hline Observations & \multicolumn{4}{|c|}{354} \\
\hline Countries & \multicolumn{4}{|c|}{20} \\
\hline
\end{tabular}


Table 2: Main results, All

\begin{tabular}{|c|c|c|c|c|c|c|}
\hline & \multicolumn{3}{|c|}{ OLS estimates } & \multicolumn{3}{|c|}{ FE estimates } \\
\hline & (i) & (ii) & (iii) & (i) & (ii) & (iii) \\
\hline Start-up incentives & $\begin{array}{c}0.9978^{*} \\
(0.5080)\end{array}$ & $\begin{array}{c}0.8977^{*} \\
(0.5100)\end{array}$ & $\begin{array}{c}0.8032^{* *} \\
(0.3120)\end{array}$ & $\begin{array}{l}0.9978^{*} \\
(0.4934)\end{array}$ & $\begin{array}{c}0.8977^{*} \\
(0.4950)\end{array}$ & $\begin{array}{c}0.7833^{* *} \\
(0.3082)\end{array}$ \\
\hline Employment rehabilitation & & $\begin{array}{c}-0.0549 \\
(0.2152)\end{array}$ & $\begin{array}{l}-0.1563 \\
(0.1920)\end{array}$ & & $\begin{array}{c}-0.0549 \\
(0.2088)\end{array}$ & $\begin{array}{l}-0.1657 \\
(0.1884)\end{array}$ \\
\hline Job Creation & & $\begin{array}{c}0.0115 \\
(0.1175)\end{array}$ & $\begin{array}{c}0.0848 \\
(0.1124)\end{array}$ & & $\begin{array}{c}0.0115 \\
(0.1141)\end{array}$ & $\begin{array}{c}0.0808 \\
(0.1089)\end{array}$ \\
\hline Training & & $\begin{array}{l}-0.0867 \\
(0.0733)\end{array}$ & $\begin{array}{c}-0.0701 \\
(0.0614)\end{array}$ & & $\begin{array}{c}-0.0867 \\
(0.0712)\end{array}$ & $\begin{array}{c}-0.0718 \\
(0.0590)\end{array}$ \\
\hline Employment incentives & & $\begin{array}{c}-0.1652 \\
(0.1007)\end{array}$ & $\begin{array}{l}-0.0065 \\
(0.0755)\end{array}$ & & $\begin{array}{l}-0.1652 \\
(0.0978)\end{array}$ & $\begin{array}{c}0.0099 \\
(0.0750)\end{array}$ \\
\hline Early retirement & & $\begin{array}{l}-0.1155 \\
(0.1354)\end{array}$ & $\begin{array}{c}-0.1456 \\
(0.1024)\end{array}$ & & $\begin{array}{c}-0.1155 \\
(0.1314)\end{array}$ & $\begin{array}{l}-0.1329 \\
(0.1015)\end{array}$ \\
\hline Out-of-work support & & $\begin{array}{c}0.0148 \\
(0.0252)\end{array}$ & $\begin{array}{c}-0.0506^{* *} \\
(0.0212)\end{array}$ & & $\begin{array}{c}0.0148 \\
(0.0245)\end{array}$ & $\begin{array}{c}-0.0533^{* * *} \\
(0.0199)\end{array}$ \\
\hline Union coverage & & & $\begin{array}{c}0.0002 \\
(0.0013)\end{array}$ & & & $\begin{array}{c}0.0003 \\
(0.0012)\end{array}$ \\
\hline Union density & & & $\begin{array}{c}-0.0068^{*} \\
(0.0035)\end{array}$ & & & $\begin{array}{c}-0.0062^{* *} \\
(0.0031)\end{array}$ \\
\hline Unit labour Cost & & & $\begin{array}{l}-0.0005 \\
(0.0032)\end{array}$ & & & $\begin{array}{l}-0.0007 \\
(0.0031)\end{array}$ \\
\hline Unemployment rate & & & $\begin{array}{c}0.0192^{* * * *} \\
(0.0041)\end{array}$ & & & $\begin{array}{c}0.0196^{* * *} \\
(0.0040)\end{array}$ \\
\hline EPL regular contracts & & & $\begin{array}{l}-0.0040 \\
(0.0337)\end{array}$ & & & $\begin{array}{c}0.0155 \\
(0.0318)\end{array}$ \\
\hline EPL temporary contracts & & & $\begin{array}{l}-0.0152 \\
(0.0142)\end{array}$ & & & $\begin{array}{l}-0.0133 \\
(0.0141)\end{array}$ \\
\hline Observations & 354 & 354 & 354 & 354 & 354 & 354 \\
\hline Clusters & 20 & 20 & 20 & 20 & 20 & 20 \\
\hline R-squared & 0.980 & 0.982 & 0.986 & 0.5663 & 0.5990 & 0.6927 \\
\hline
\end{tabular}

Note: The dependent variable is the logarithm of self-employment rate, as a proportion of civilian employment. All specifications include country and year fixed effects. Standard errors are heteroskedasticity robust and clustered at the country level. 
Table 3: Main results, Women vs Men

\begin{tabular}{|c|c|c|c|c|c|c|}
\hline & \multicolumn{3}{|c|}{ OLS estimates } & \multicolumn{3}{|c|}{ FE estimates } \\
\hline & (i) & (ii) & (iii) & (i) & (ii) & (iii) \\
\hline \multirow[t]{2}{*}{ Start-up incentives } & 0.1198 & $0.5520^{*}$ & $0.5138^{*}$ & 0.1198 & $0.5520^{* *}$ & 0.3367 \\
\hline & $(0.3025)$ & $(0.2693)$ & $(0.2551)$ & $(0.2934)$ & $(0.2610)$ & $(0.6946)$ \\
\hline \multirow[t]{2}{*}{ Employment rehabilitation } & & 0.2145 & 0.1887 & & 0.2145 & -0.2308 \\
\hline & & $(0.1850)$ & $(0.1604)$ & & $(0.1793)$ & $(0.3300)$ \\
\hline \multirow[t]{2}{*}{ Job Creation } & & -0.0869 & -0.0473 & & -0.0869 & -0.5966 \\
\hline & & $(0.1030)$ & $(0.0719)$ & & $(0.0998)$ & $(0.4023)$ \\
\hline \multirow[t]{2}{*}{ Training } & & $-0.1891^{* *}$ & $-0.1627^{* * *}$ & & $-0.1891^{* *}$ & $-0.8182^{* * *}$ \\
\hline & & $(0.0683)$ & $(0.0552)$ & & $(0.0662)$ & $(0.2298)$ \\
\hline \multirow[t]{2}{*}{ Employment incentives } & & $-0.2058 * *$ & -0.0848 & & $-0.2058 * *$ & 0.0529 \\
\hline & & $(0.0962)$ & $(0.0828)$ & & $(0.0932)$ & $(0.1746)$ \\
\hline \multirow[t]{2}{*}{ Early retirement } & & 0.1631 & 0.0451 & & 0.1631 & $0.4200^{* * *}$ \\
\hline & & $(0.1287)$ & $(0.0935))$ & & $(0.1248)$ & $(0.1292)$ \\
\hline \multirow[t]{2}{*}{ Out-of-work support } & & -0.0012 & -0.0010 & & -0.0012 & 0.1250 \\
\hline & & $(0.0236)$ & $(0.0262)$ & & $(0.0229)$ & $(0.0799)$ \\
\hline \multirow[t]{2}{*}{ Union coverage } & & & -0.001 & & & -0.0027 \\
\hline & & & $(0.0009)$ & & & $(0.0023)$ \\
\hline \multirow[t]{2}{*}{ Union density } & & & -0.0033 & & & $-0.0047^{* * *}$ \\
\hline & & & $(0.0030)$ & & & $(0.0016)$ \\
\hline \multirow[t]{2}{*}{ Unit labour Cost } & & & -0.0018 & & & $-0.0288^{* *}$ \\
\hline & & & $(0.0024)$ & & & $(0.0128)$ \\
\hline \multirow[t]{2}{*}{ Female unemployment rate } & & & $0.0198 * * *$ & & & 0.0065 \\
\hline & & & $(0.0057)$ & & & $(0.0168)$ \\
\hline \multirow[t]{2}{*}{ Male unemploymeny rate } & & & $-0.0251^{* * *}$ & & & -0.0373 \\
\hline & & & $(0.0085)$ & & & $(0.0308)$ \\
\hline \multirow[t]{2}{*}{ EPL regular contracts } & & & $0.0893^{* * *}$ & & & $0.0953^{* * *}$ \\
\hline & & & $(0.0276)$ & & & $(0.0305)$ \\
\hline \multirow{2}{*}{ EPL temporary contracts } & & & -0.0008 & & & 0.0570 \\
\hline & & & $(0.0149)$ & & & $(0.0553)$ \\
\hline Observations & 340 & 340 & 340 & 340 & 340 & 340 \\
\hline Clusters & 20 & 20 & 20 & 20 & 20 & 20 \\
\hline R-squared & 0.9664 & 0.9730 & 0.9786 & 0.4189 & 0.5340 & 0.2680 \\
\hline
\end{tabular}

Note: The dependent variable is the ratio between female and male self-employment. All specifications include country and year fixed effects. Standard errors are heteroskedasticity robust and clustered at the country level. 
Table 4: IV results

\begin{tabular}{|c|c|c|c|c|}
\hline & \multicolumn{2}{|l|}{ All } & \multicolumn{2}{|c|}{ Women vs men } \\
\hline & First stage & Second stage & First stage & Second stage \\
\hline Start-up incentives & & $\begin{array}{c}1.7949^{* *} \\
(0.9162)\end{array}$ & & $\begin{array}{c}2.2065^{* * *} \\
(0.7846)\end{array}$ \\
\hline Ideology & $\begin{array}{c}0.0522^{* * *} \\
(0.0114)\end{array}$ & & $\begin{array}{c}0.0573^{* * *} \\
(0.0120)\end{array}$ & \\
\hline Employment rehabilitation & $\begin{array}{c}-0.1067^{* * *} \\
(0.025)\end{array}$ & $\begin{array}{l}-0.0517 \\
(0.1249)\end{array}$ & $\begin{array}{c}-0.0951^{* * *} \\
(0.0343)\end{array}$ & $\begin{array}{c}0.3477 \\
(0.1294)\end{array}$ \\
\hline Job Creation & $\begin{array}{c}0.0018 \\
(0.0146)\end{array}$ & $\begin{array}{l}0.0856^{*} \\
(0.0487)\end{array}$ & $\begin{array}{c}0.0060 \\
(0.0172)\end{array}$ & $\begin{array}{l}-0.0483 \\
(0.0506)\end{array}$ \\
\hline Training & $\begin{array}{c}0.0088 \\
(0.0133)\end{array}$ & $\begin{array}{l}-0.078^{*} \\
(0.0412)\end{array}$ & $\begin{array}{c}0.0228^{*} \\
(0.0129)\end{array}$ & $\begin{array}{c}-0.1962^{* * *} \\
(0.0377)\end{array}$ \\
\hline Employment incentives & $\begin{array}{c}0.0744^{* * *} \\
(0.0191)\end{array}$ & $\begin{array}{c}-0.0754 \\
(0.0799)\end{array}$ & $\begin{array}{c}0.0547^{* * *} \\
(0.0198)\end{array}$ & $\begin{array}{c}-0.1655^{* *} \\
(0.0708)\end{array}$ \\
\hline Early retirement & $\begin{array}{c}-0.0219 \\
(0.0186)\end{array}$ & $\begin{array}{c}-0.1260^{* *} \\
(0.0554)\end{array}$ & $\begin{array}{c}-0.0086 \\
(0.0221)\end{array}$ & $\begin{array}{c}0.0485 \\
(0.0607)\end{array}$ \\
\hline Out-of-work support & $\begin{array}{c}0.0165^{* * *} \\
(0.0045)\end{array}$ & $\begin{array}{c}-0.0675^{* * *} \\
(0.0205)\end{array}$ & $\begin{array}{c}0.0104^{*} \\
(0.0057)\end{array}$ & $\begin{array}{c}-0.0174 \\
(0.0149)\end{array}$ \\
\hline Union coverage & $\begin{array}{c}0.0006^{* * *} \\
(0.0002)\end{array}$ & $\begin{array}{c}-0.0007 \\
(0.0011)\end{array}$ & $\begin{array}{c}0.0006^{* * *} \\
(0.0002)\end{array}$ & $\begin{array}{c}-0.0024^{* *} \\
(0.0009)\end{array}$ \\
\hline Union density & $\begin{array}{c}-0.0019 * * * \\
(0.0006)\end{array}$ & $\begin{array}{c}-0.0047^{*} \\
(0.0027)\end{array}$ & $\begin{array}{c}-0.0021^{* * *} \\
(0.0007)\end{array}$ & $\begin{array}{c}0.0068^{* *} \\
(0.003)\end{array}$ \\
\hline Unit labour Cost & $\begin{array}{c}0.0002 \\
(0.0004)\end{array}$ & $\begin{array}{c}-0.0008 \\
(0.0017)\end{array}$ & $\begin{array}{c}0.0004 \\
(0.0004)\end{array}$ & $\begin{array}{c}-0.0026 \\
(0.0018)\end{array}$ \\
\hline Unemployment rate & $\begin{array}{c}-0.001 \\
(0.0009)\end{array}$ & $\begin{array}{c}0.0203^{* * *} \\
(0.0024)\end{array}$ & & \\
\hline Female Unemployment rate & & & $\begin{array}{c}-0.0015 \\
(0.0012)\end{array}$ & $\begin{array}{c}0.0233^{* * *} \\
(0.0044\end{array}$ \\
\hline Male Unemployment rate & & & $\begin{array}{c}0.0017 \\
(0.0021)\end{array}$ & $\begin{array}{c}-0.0288^{* * *} \\
(0.0061)\end{array}$ \\
\hline EPL regular contracts & $\begin{array}{c}0.0481 * * * \\
(0.0167)\end{array}$ & $\begin{array}{c}-0.048123 \\
(0.0519)\end{array}$ & $\begin{array}{c}0.0526 * * * \\
(0.0172)\end{array}$ & $\begin{array}{c}0.0053 \\
(0.0491)\end{array}$ \\
\hline EPL temporary contracts & $\begin{array}{c}-0.0066^{* * *} \\
(0.0024) \\
\end{array}$ & $\begin{array}{c}-0.0066 \\
(0.0109) \\
\end{array}$ & $\begin{array}{c}0.0073^{* * *} \\
(0.0027) \\
\end{array}$ & $\begin{array}{c}0.0155 \\
(0.0105) \\
\end{array}$ \\
\hline Observations & 354 & 354 & 340 & 340 \\
\hline Clusters & 20 & 20 & 20 & 20 \\
\hline R-squared & 0.4825 & 0.6670 & 0.4384 & 0.5186 \\
\hline$F$ test of excluded instruments & $\mathrm{F}(1,304)=21.09$ & & $\mathrm{~F}(1,289)=22.68$ & \\
\hline
\end{tabular}

Note: All specifications are panel IVFE regressions. Standard errors are heteroskedasticity robust and clustered at the country level. 